\title{
Risk factors involved in the onset of postoperative delirium after transurethral prostatectomy in elderly- a retrospective study
}

\section{Savu Carmen ${ }^{1}$, Burchiu Eliza ${ }^{1}$, Surcel C ${ }^{2,3}$, Mirvald C ${ }^{2,3}$, Mihai V', Pavelescu C ${ }^{2}$, Tulbure Ruxanda \\ ${ }^{1}$ Anestesiology and Intensive Care Unit, Fundeni Clinical Institute, Bucharest, Romania \\ ${ }^{2}$ Centre of Uronephrology and Renal Transplantation, Fundeni Clinical Institute, Bucharest, Romania. \\ 3 "Carol Davila" University of Medicine and Pharmacy, Bucharest, Romania.}

\section{Introduction:}

- The aim of this study was to investigate the incidence of postoperative delirium (POD) in elderly patients undergoing monopolar TURP for benign prostatic hyperplasia (BPH) and to identify the risk factors involved in the onset and severity of POD.

- The elderly represent a large cohort of patients undergoing urological procedures in which transurethral prostate resection (TURP) is one of the most frequently performed.

\section{Material and method:}

- 240 patients aged over 70 years underwent elective surgery for BPH in our Department between Jan 2013- Dec 2015.

- In all cases, monopolar transurethral prostatectomy (TURP) was performed. Distilled water was used as rinsing liquid.

- Patients were evaluated preoperatively, immediately postoperative and at 5 days after TURP.

- Inclusion criteria:

- spinal anesthesia;

- preoperative minimental state examination (MMSE) $>27$

- Patients with psychiatric disorders, preoperative antidepressants, or who developed postTUR syndrome were excluded.

- Postoperative delirium was diagnosed using the Confusion Assessment method (CAM).

- After surgery, patients were divided into 3 groups based on POD severity:

- Group 1- no delirium - $217(90.41 \%)$ patients

- Group 2 - POD for 2-3 days - $17(7.08 \%)$ patients

- Group $3-\mathrm{POD}>4$ days $-10(4.16 \%)$ patients

- Perioperative data included operating time, TAM, HR, SpO2, rectal temperature, blood loss, volume of rinsing liquid.

- POD treatment consisted in titrated Haloperidol ( $0.5 \mathrm{mg}$ i.v at a time); pain management and non-pharmacological measures: early nutrition, assist with the sleep-wake cycle, presence of family at the bedside, glasses and hearing aid, etc.

- Benzodiazepines were used if sedation was required.

- For comparison between these 3 groups we used Mann-Whitney $U$ test for continuous data, Kruskall Wallis test for non parametric variables and $x 2$ test for ordinal data. A $p$ value $<0.05$ was considered statistically significant.

\section{Results:}

\begin{tabular}{|c|c|c|c|c|c|c|c|c|c|c|c|}
\hline \multicolumn{3}{|l|}{ Demographic data } & \multicolumn{3}{|c|}{ Total } & \multicolumn{2}{|c|}{ Group 1} & \multicolumn{2}{|r|}{ Group 2} & Group 3 & $\mathbf{p}$ \\
\hline \multirow{2}{*}{\multicolumn{3}{|c|}{$\begin{array}{l}\text { Age (mean, } \mathrm{Cl} \text { ) } \\
\text { Prostate volume, cc (mean, } \mathrm{Cl})\end{array}$}} & \multicolumn{3}{|c|}{$76.3(70-87)$} & \multicolumn{2}{|c|}{$75.2(70-87)$} & \multicolumn{2}{|r|}{$76.1(71-81)$} & $74.8(70-85)$ & 0.474 \\
\hline & & & \multirow{2}{*}{\multicolumn{3}{|c|}{$\begin{array}{c}65.22(25.6-85.4) \\
54(22.5)\end{array}$}} & \multicolumn{2}{|c|}{$60.21(26.33-76.51)$} & \multicolumn{2}{|c|}{$62.47(25.6-81.44)$} & $64.21(26.6-85.4)$ & 0.321 \\
\hline \multicolumn{3}{|c|}{ History of $\uparrow$ alcohol intake No. (\%) } & & & & \multicolumn{2}{|c|}{$32(15.02)$} & \multicolumn{2}{|c|}{$11(64.7)$} & $6(60)$ & $<0.001$ \\
\hline \multicolumn{3}{|c|}{ History of smoking No. (\%) } & \multicolumn{3}{|c|}{$73(30.41)$} & \multicolumn{2}{|c|}{$24(11.26)$} & \multicolumn{2}{|r|}{$6(35.29)$} & $4(40)$ & 0.032 \\
\hline \multicolumn{3}{|c|}{ Preop. urinary catheter No. (\%) } & \multicolumn{3}{|c|}{$14(5.83)$} & \multirow{2}{*}{\multicolumn{2}{|c|}{$\begin{array}{l}3(1.4) \\
3(2-4)\end{array}$}} & & $7(41.17)$ & $4(40)$ & $<0.001$ \\
\hline \multicolumn{3}{|c|}{ ASA score (mean, $\mathrm{Cl}$ ) } & \multicolumn{3}{|c|}{$3(2-4)$} & & & & $3(2-4)$ & $3(2-4)$ & 0.214 \\
\hline Marital status - single No & $(\%)$ & & & $31(12.91$ & & $12(5.63$ & & & $12(70.58)$ & $7(70)$ & 0.032 \\
\hline Preoperative $\mathrm{Hb}$ (mean, & & & 11.4 & $4(8.75-1$ & 15.17) & $11.1(9.44-1$ & 4.78) & 10.7 & $8(8.75-13.45)$ & $11.24(9.31-15.17)$ & 0.421 \\
\hline Previous surgery (No, $\%$ & & & & $28(11.6$ & & $19(8.9$ & & & $6(35.29)$ & $2(20)$ & 0.15 \\
\hline Preop MMSE (points), me & ean & & & $28(27-3 c$ & & $29(27-3$ & & & $28(27-30)$ & $28(27-30)$ & 0.345 \\
\hline Perioperative data & & & & Total & & Group & & & Group 2 & Group 3 & $\mathbf{p}$ \\
\hline Operative time, $\min$, (mea & $\mathrm{n}, \mathrm{Cl})$ & & & $6.21(35-$ & $5-104)$ & $48.35(42$ & $-71)$ & & $1.14(35-94)$ & $58.22(41-104)$ & 0.042 \\
\hline Mean Rectal temperature & $\left({ }^{\circ} \mathrm{C}\right)$ & & & $3.8(35.1-3$ & $-37.2)$ & $37(35.1-3$ & 37.2) & & $.8(35.1-37.2)$ & $36.6(35.1-37.2)$ & 0.032 \\
\hline Hypothermia time, min, (me & an, $\mathrm{Cl}$ ) & & & $39.64(20$ & $0-84)$ & $33.14(20$ & $-41)$ & & $6.45(20-74)$ & $52.36(30-84)$ & 0.005 \\
\hline Rinsing liquid (L), mean & $\mathrm{Cl}$ & & & $.21(12.2-$ & 2-38.6) & $17.54(12.4$ & $-27.2)$ & 19. & $75(13.1-37.8)$ & $18.08(12.2-38.6)$ & 0.122 \\
\hline Blood loss (g), mean, & & & & $14(0.4-4$ & $-4.21)$ & $1.61(0.4-$ & 1.92) & & $.41(1.1-3.6)$ & $2.12(0.6-4.21)$ & 0.032 \\
\hline Postoperative data & To & & Grou & up 1 & Group 2 & Group 3 & $\mathbf{p}$ & & & & \\
\hline Hospital stay (days) mean, $\mathrm{Cl}$ & $7.1(3$ & -21) & $5.4(3$ & $3-10)$ & $7.3(5-12)$ & $12.6(6-21)$ & $<0.004$ & & & & \\
\hline ICU stay (days) mean, CI & $3(0$ & & $1(0-$ & & $3.14(2-4)$ & $6.51(4-7)$ & $<0.004$ & & & & \\
\hline Complication rates Clavien scale & $(\mathrm{No}, \%)$ & & & & & & & & & & \\
\hline I & $29(12$ & $2.08)$ & $20(9$. & .38) & $7(41.17)$ & $2(20)$ & 0.24 & & & & \\
\hline II & $27(11$ & $.25)$ & $0(0$ & & $17(100)$ & $10(100)$ & 0.1 & & & & \\
\hline Illa & $5(2$. & 08) & $0(0$ & & $4(23.52)$ & $1(10)$ & 0.98 & 37.5 & & & \\
\hline$>$ IIla & 0( & & $0(0$ & & $0(0)$ & $0(0)$ & $\mathrm{n} / \mathrm{a}$ & & & & \\
\hline Blood Transfusion No (\%) & $2(0$ & 83) & $0(0$ & & $1(5.82)$ & $1(10)$ & $\mathrm{n} / \mathrm{a}$ & 37 & & & \\
\hline & & Univa & ariate & & & Multivariate & & $\stackrel{\bar{\Xi}}{\text { ข }}_{36.5}$ & & & \\
\hline & HR & & $5 \% \mathrm{Cl}$ & $\mathbf{p}$ & HR & $95 \% \mathrm{Cl}$ & $\mathbf{p}$ & & & & \\
\hline Age (continuous variable) & & & & & & & & & & & \\
\hline$<76$ (reference) & 1.000 & & & & 1.000 & & & & & & \\
\hline$>76$ & 2.049 & 1.72 & $2-2.596$ & $<0.0001$ & 1.794 & $1.7255-2.8999$ & $<0.0001$ & & & & - TNO PUD \\
\hline Marital status-single & & & & & & & & 胥 35.5 & & & - Moderate POD \\
\hline No & 1.266 & 1.18 & $7-1.351$ & 0.04 & 1.2331 & $0.610-1.302$ & 0.018 & & & & $\neg$ Severe POD \\
\hline Yes & 3.247 & 2.70 & $3-3.900$ & $<0.0001$ & 2.5963 & $1.9142-3.5214$ & $<0.0001$ & 35 & & & \\
\hline History of $\uparrow$ alcohol intake & 1.23 & 1.15 & $9-1.575$ & 0.004 & 1.6279 & $1.207-2.196$ & 0.003 & & & & \\
\hline $\begin{array}{l}\text { Preoperative urinary } \\
\text { catheterization }\end{array}$ & 1.914 & 1.63 & $7-2.438$ & $<0.0001$ & 1.4172 & $1.1946-1.6814$ & $<0.005$ & 34.5 & & & \\
\hline MMSE (ordinal) & 1.93 & 1.45 & $9-2.575$ & 0.68 & 1.6279 & $1.207-2.196$ & 0.34 & 34 & & & \\
\hline Blood loss $>2$ g/dL & 2.13 & 1.25 & $9-3.575$ & 0.214 & 1.921 & $1.217-2.396$ & 0.117 & & $\begin{array}{lllll}0 & 10 & 20 & 30 & 40\end{array}$ & 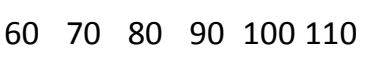 & \\
\hline Rectal temperature $<36$ & & & & & & & & & & time $(\min )$ & \\
\hline$<40 \mathrm{~min}$ & 1.821 & 0.46 & $6-7.115$ & 0.3890 & 1.369 & $0.481-2.306$ & 0.284 & & & & \\
\hline$>40$ min & 5.06 & 3.89 & $5-6.575$ & $<0.0001$ & 3.2095 & $2.395-4.302$ & 0.0322 & & & & \\
\hline
\end{tabular}

\section{Conclusions:}

Age $>76$, marital status, alcohol use disorders, preoperative urinary catheterization were found to be preoperative predictors for onset of POD, while hipothermia time was associated with POD severity.

- Reducing the operative time, usage of preheated rinsing liquids in association with specific treatment may decrease POD incidence, morbidity and hospitalization costs. 